03

\title{
О количественной оценке степени независимости преобразования кубитов квантовым вентилем или каналом*
}

\author{
(С) М.П. Фалеева ${ }^{1}$, И.Ю. Попов ${ }^{1, \uparrow, ~ И . ~ Ж е ж у л а ~}{ }^{2}$ \\ ${ }^{1}$ Университет ИТМО, \\ 197101 Санкт-Петербург, Россия \\ ${ }^{2}$ Университет Сафарика, \\ 04180 Кошице, Словакия \\ ฯ e-mail: popov1955@gmail.com
}

Поступила в редакцию 22.12.2017 г.

Рассмотрен многокубитовый канал (квантовый вентиль). Предложена процедура вычисления расстояния от матрицы квантового вентиля до подпространства матриц, являющихся тензорными произведениями матриц преобразования подсистем кубитов. Величина данного расстояния отражает степень независимости преобразования данных подсистем кубитов. Рассмотрено применение предложенного подхода к волноводной реализации квантовых битов.

DOI: $10.21883 /$ OS.2018.05.45953.313-17

\section{Введение}

Перепутанность - важнейшее свойство квантовой системы, позволяющее реализовать квантовые алгоритмы [1]. При этом очень важно знать, преобразуются ли отдельные кубиты при выполняемых многокубитовых операциях независимо. Достаточным условием такой независимости является возможность представления матрицы квантового вентиля (канала) в виде тензорного произведения матриц преобразования отдельных кубитов. В противном случае возникает вопрос о мере степени независимости преобразования кубитов, которая позволяла бы сравнивать с этой точки зрения каналы между собой. В качестве такой меры предлагается использовать расстояние от матрицы данного вентиля до подпространства матриц, являющихся тензорными произведениями. Рассматриваемый вопрос очень важен для описания многокубитовых квантовых каналов, где матрица преобразования кубитов каналом играет роль матрицы квантового вентиля. Интерес к данной задаче усилился в последнее время в связи с изучением вопроса о создании квантовых каналов через свободное пространство (см., например, недавние эксперименты по телепортации запутанности [2]). Если независимости преобразования кубитов нет, то при передачи может возникнуть изменение степени перепутанности, что нарушит правильную работу канала. Этот вопрос также важен при анализе устойчивости квантовых алгоритмов (например, [3-5]). Следует отметить, что степень независимости преобразования кубитов отражает несколько другое свойство канала, отличающееся от обычно используемой точности воспроизведения запутанности [6,7]. Это показывает, в частности, простой пример оператора SWAP, который не меняет запутанность, но не преобразует кубиты независимо.

\footnotetext{
* Х Международная конференция молодых ученых и специалистов „Оптика 2017“, 16-20 октября 2017 г., Санкт-Петербург, Россия
}

Указанный выше параметр и предложенная в настоящей работе процедура его определения могут быть полезны и в других задачах. Если в качестве рассматриваемой матрицы взять матрицу плотности многокубитового состояния, то ее расстояние от подпространства матриц плотности, являющихся матрицами плотности отдельных кубитов, будет мерой перепутанности данного многокубитового состояния [8-10]. Описанное расстояние возникает и во многих других вопросах, связанных с квантовым преобразованием информации, таких как квантовые процессы [11], квантовая криптография [12], квантовые фазовые переходы [13], квантовый предел скорости [14], емкость квантового канала [15], квантовая перепутанность и когерентность [16-18], запись в квантовую память [19]. Математические аспекты вопроса освещены в [20,21]. Рассматриваемое в работе расстояние связано с известной теоремой ЭккартаЯнга-Мирского [22], описывающей приближение матриц матрицами меньшего ранга. В частности, мы предлагаем модификацию этой теоремы и иную процедуру вычисления расстояния. Мы используем разложение по сингулярным числам [23,24]. Интересно, что подобная задача может возникнуть и в классической компьютерной науке, например, при сравнении кодов [25].

\section{Основной результат}

Введем оператор векторизации $v e c$, который переводит матрицу $M$ в вектор vec $M$ по следующему правилу:

$$
\operatorname{vec} M=\left(m_{1}^{\prime}, \ldots, m_{p}^{\prime}\right)^{\prime},
$$

где векторы $m_{1}, \ldots, m_{p}$ есть столбцы матрицы $M$, штрих обозначает операцию транспонирования с комплексным сопряжением.

Возьмем две матрицы $B_{m \times m}, C_{n \times n}$ и их тензорное (кронекерово) произведение $R_{m n \times m n}=B \otimes C$. Введем 
матрицу $R$, которая получается из $R$ следующим образом:

$$
R_{m^{2} \times n^{2}}=\operatorname{vecB}(\operatorname{vec} C)^{\prime} .
$$

Ясно, что матрица $R$ состоит из тех же элементов, что и $R_{m n \times m n}=B \otimes C$, размещенных в другом порядке. Для выполнения данной операции с произвольной матрицей $A_{m n \times m n}$, разбиваем ее на блоки размера $m \times n$ и получаем $A_{m^{2} \times n^{2}}$, следуя той же процедуре, что с матрицей $R$. Основной математический результат мы сформулируем в качестве математической теоремы.

Теорема 1. Для произвольной матрицы $A_{m n \times m n}$ норма $\|A-B \otimes C\|$ минимальна, если матрицы $B_{m \times m}$ и $C_{n \times n}$ таковы, что vec $B(\text { vec } C)^{\prime}=k b c^{\prime}$. Здесь $k=\sigma_{1}$ есть максимальное из сингулярных чисел матрицы $A\left(A_{m^{2} \times n^{2}}\right)$, $b=u_{1}$ и $c=v_{1}$ - правый и левый сингулярные векторы матрицы $A$, соответствующие сингулярному числу $\sigma_{1}$.

Замечание. Что касается меры степени независимости преобразования кубитов, то следует брать нормированное расстояние: $\|A\|^{-1}\|A-B \otimes C\|$. Для унитарных матриц $p \times p:\|A\|=\sqrt{p}$.

\section{Обсуждение результатов}

В качестве первого примера рассмотрим оператор $C N O T$ в стандартном базисе. Мы найдем матрицы $B, C$ размера $2 \times 2$ такие, что их тензорное произведение наиболее близко к матрице $U_{C N O T}$ :

$$
U_{C N O T}=\left(\begin{array}{llll}
1 & 0 & 0 & 0 \\
0 & 1 & 0 & 0 \\
0 & 0 & 0 & 1 \\
0 & 0 & 1 & 0
\end{array}\right)
$$

Соответственно

$$
U_{C N O T}=\left(\begin{array}{llll}
1 & 0 & 0 & 1 \\
0 & 0 & 0 & 0 \\
0 & 0 & 0 & 0 \\
0 & 1 & 1 & 0
\end{array}\right)
$$

Для вычисления $\left\|U_{C N O T}-\sigma_{1} u_{1} v_{1}^{\prime}\right\|$ строим сингулярное разложение на основе известной теоремы (следует отметить, что разложение по сингулярным числам связано с разложением Шмидта, широко используемым в квантовой теории информации).

Теорема 2. Для любой матрицы $A_{m \times n}$, существуют унитарные матрицы $U$ и $V$ такие, что $A=U \lambda V^{\prime}$, где $A_{m \times n}-$ матрицы с ненулевыми элементами на диагонали $\lambda_{j j}=\sigma_{j},\left\{\sigma_{i}\right\}_{i=1}^{s}-$ сингулярные числа матрицы $A_{m \times n}$. Здесь $s$ - ранг $A$, матрицы $U_{m \times m}$ и $V_{n \times n}-$ две унитарные матрицы, образованные левыми $\left(\left\{u_{i}\right\}_{i=1}^{m}\right)$ и правыми $\left(\left\{v_{i}\right\}_{i=1}^{n}\right)$ сингулярными векторами. Имеет место следующее сингулярное разложение матрицы:

$$
A=\sum_{i=1}^{s} \sigma_{i} u_{i} v_{i}
$$

В случае CNOT имеем: $\sigma_{1}=\sigma_{2}=\sqrt{2}, \sigma_{3}=\sigma_{4}=0$. $\left\|U_{C N O T}-\sigma_{1} u_{1} v_{1}\right\|=\sqrt{2}$.

Рассмотрим двухкубитовый оператор $U_{S W A P}$, который отличается тем, что не порождает запутанность. Однако он не преобразует кубиты независимо. Его матрица

$$
U_{S W A P}=\left(\begin{array}{llll}
1 & 0 & 0 & 0 \\
0 & 0 & 1 & 0 \\
0 & 1 & 0 & 0 \\
0 & 0 & 0 & 1
\end{array}\right)
$$

В этом случае получается $U_{S W A P}=U_{S W A P}, \sigma_{1}=\sigma_{2}=$ $=\sigma_{3}=\sigma_{4}=1,\left\|U_{S W A P}-\sigma_{1} u_{1} v_{1}^{\prime}\right\|=\sqrt{3}$.

Рассмотрим матрицу плотности для стандартного двухкубитового чистого состояния:

$$
|\psi>=a| 00>+b|01>+c| 10>+d \mid 11>,
$$

где $|a|^{2}+|b|^{2}+|c|^{2}+|d|^{2}=1$. Критерий незапутанности в этом случае $\delta=a d-b c=0$. Значение $|a d-b c|$ можно рассматривать как меру запутанности. Матрица плотности $S$ такова: $S=|\psi><\psi|$. Следуя нашей процедуре, получаем

$$
S^{*} S=\left[\begin{array}{ll}
A\left[\begin{array}{ll}
A & \bar{B} \\
B & C
\end{array}\right] & B\left[\begin{array}{ll}
A & \bar{B} \\
B & C
\end{array}\right] \\
\bar{B}\left[\begin{array}{ll}
A & \bar{B} \\
B & C
\end{array}\right] & C\left[\begin{array}{ll}
A & \bar{B} \\
B & C
\end{array}\right]
\end{array}\right]=\left[\begin{array}{ll}
A & B \\
\bar{B} & C
\end{array}\right] \otimes\left[\begin{array}{ll}
A & \bar{B} \\
B & C
\end{array}\right],
$$

где $A=|a|^{2}+|b|^{2}, C=|c|^{2}+|d|^{2}, B=a \bar{c}+b \bar{d}, \bar{B}=\bar{c} a+$ $+d \bar{b}$. Соответственно собственные числа $\sigma_{i}$ матрицы $4 \times 4$ получаются как произведения собственных чисел $\lambda_{j}$ матриц $2 \times 2$ :

$$
\begin{aligned}
\lambda_{1,2} & =2^{-1} \pm \sqrt{4^{-1}-A C+|B|^{2}} \\
& =2^{-1} \pm \sqrt{4^{-1}-|a d-b c|^{2}}
\end{aligned}
$$

Соответственно получаем расстояние $\rho$ от подпространства незапутанных состояний:

$$
\begin{gathered}
\rho=1-\frac{\left|\sigma_{1}\right|^{4} C_{1}+2 \delta^{4}-\delta^{2}\left(\sigma_{1}^{2}+\overline{\sigma_{1}^{2}}\right) C_{2}}{C_{2}\left(\left|\sigma_{1}\right|^{4}+\delta\right)-2 \delta^{2}\left(\sigma_{1}^{2}+\overline{\sigma_{1}^{2}}\right)}, \\
C_{1}=A^{4}+C^{4}+4|B|^{2}\left(A C+C^{2}+A^{2}\right)+2|B|^{4}, \\
C_{2}=A^{2}+C^{2}+2|B|^{2} .
\end{gathered}
$$

Естественно, для $\delta=0$ имеем $\rho=0$, и наоборот, т.е. наблюдается связь с обычно используемой мерой запутанности.

Рассмотрим применение нашего подхода к задаче о волноводной реализации кубитов (например, [26]). Кубит представляется электронным состоянием (волной) в двух слабо связанных (через малое отверстие) квантовых волноводах. Положение электрона в первом волноводе соответствует „, $0^{“,}$, во втором - „1“. Из-за связи волноводов имеем суперпозицию состояний. Рассмотрим систему двух кубитов, т. е. четырех волноводов 


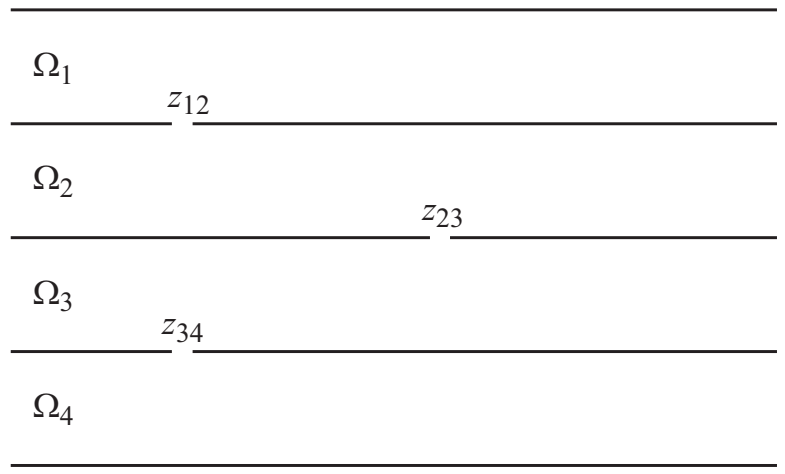

Рис. 1. Геометрия системы для волноводной реализации двух кубитов: $\Omega_{i}-$ квантовый волновод, $z_{i j}-$ отверстие связи волноводов $\Omega_{i}$ и $\Omega_{j}$.

(рис. 1). Вентиль в данном случае моделируется окном связи $z_{23}$ (в зависимости от параметров этой связи можем получать различные вентили). Для описания системы используем модель щелей нулевой ширины, в которой малые отверстия заменяются на точечные по специальной процедуре в духе модели потенциалов нулевого радиуса [27-29]. Этот подход базируется на теории самосопряженных расширений симметрических операторов [30]. В общих чертах схема такова. Исходный самосопряженный оператор - ортогональная сумма неймановских лапласианов для отдельных волноводов. Мы сужаем оператор на множество функций, обнуляющихся в выбранных граничных точках. Полученный оператор симметричен и имеет конечные индексы дефекта. Дефектными элементами являются функции Грина для волноводов с источниками в выбранных нами точках. Модельный оператор получается как самосопряженное расширение данного симметрического оператора. Мы выбираем наиболее естественное расширение, в область определения которого входят функции, имеющие непрерывную регулярную часть и отличающиеся по знаку (с двух сторон от отверстия) сингулярности. Найдем в рамках модели матрицу преобразования кубитов аналитически.

Рассмотрим одномодовый режим волноводов. Решение модельной задачи рассеяния имеет вид

$\begin{cases}\psi_{1}(z, k)+a_{12} G_{1}\left(z, z_{12}, k\right), & z \in \Omega_{1}, \\ \psi_{2}(z, k)-a_{12} G_{2}\left(z, z_{12}, k\right)+a_{23} G_{2}\left(z, z_{23}, k\right), & z \in \Omega_{2}, \\ \psi_{3}(z, k)+a_{34} G_{3}\left(z, z_{34}, k\right)-a_{23} G_{3}\left(z, z_{23}, k\right), & z \in \Omega_{3}, \\ \psi_{4}(z, k)-a_{34} G_{3}\left(z, z_{34}, k\right), & z \in \Omega_{4} .\end{cases}$

Здесь $\psi_{i}$ - приходящая волна в $\Omega_{i}, z=\left(\begin{array}{l}x \\ y\end{array}\right)$. Волноводы считаются одинаковыми. Соответственно одинаковы и функции Грина $G_{i}$. Условия согласования в точках связи дают

$\left\{\begin{array}{l}\psi_{1}\left(z_{12}, k\right)+a_{12} g=\psi_{2}\left(z_{12}, k\right)+a_{23} G-a_{12} g, \\ \psi_{2}\left(z_{23}, k\right)-a_{12} G+a_{23} g=\psi_{3}\left(z_{23}, k\right)-a_{34} G-a_{23} g, \\ \psi_{3}\left(z_{34}, k\right)+a_{34} g-a_{23}=\psi_{4}\left(z_{34}, k\right)-a_{34} g .\end{array}\right.$

Здесь

$$
\begin{gathered}
G=G_{i}\left(z_{23}, z_{34}, k\right), \\
g=\lim _{x \rightarrow x_{j, j+1}}\left(G_{i}\left(z, z_{j, j+1}, k\right)-G_{i}\left(z, z_{j, j+1}, k_{0}\right)\right),
\end{gathered}
$$

$k_{0}$ - модельный параметр, который характеризует силу связи через отверстие, $k_{0}^{2}<0$. Из-за симметрии $g$ и $G$ не зависят от $i, j$. Решения системы (2), $a_{j, j+1}$, подставляются в (1). Учитывая известное выражение функции Грина для волновода, получаем уходящие волны $\psi_{i}^{\text {out }}$. Они связаны с приходящими волнами $\psi_{i}$ матрицей преобразования $T$ :

$$
\left[\begin{array}{l}
\psi_{1}^{\text {out }} \\
\psi_{2}^{\text {out }} \\
\psi_{3}^{\text {out }} \\
\psi_{4}^{\text {out }}
\end{array}\right]=T\left[\begin{array}{l}
\psi_{1} \\
\psi_{2} \\
\psi_{3} \\
\psi_{4}
\end{array}\right]
$$

Таким образом, мы находим матрицу преобразования. Ее элементы таковы:

$$
\begin{aligned}
& t_{11}=1+c_{1} c_{2}(2 k)^{-1}, \quad t_{12}=-c_{1} c_{2}(2 k)^{-1}, \\
& t_{13}=c_{1}(2 k)^{-1}, \quad t_{14}=-c_{1}(2 k)^{-1}, \\
& t_{21}=\left(-c_{1} c_{2}+\frac{\exp ^{-i k L}\left(1+2 g c_{1} c_{2}\right)}{G}\right)(2 k)^{-1}, \\
& t_{22}=1-\left(-c_{1} c_{2}+\frac{\exp ^{-i k L}\left(1+2 g c_{1} c_{2}\right)}{G}\right)(2 k)^{-1}, \\
& t_{23}=c_{1}\left(-1+\frac{2 g}{G} \exp ^{-i k L}\right)(2 k)^{-1}, \\
& t_{24}=-c_{1}\left(-1+\frac{2 g}{G} \exp ^{-i k L}\right)(2 k)^{-1}, \\
& t_{31}=\left(-\frac{1+2 g c_{1} c_{2}}{G} \exp ^{-i k L}+\frac{2 g}{G}+c_{1} c_{2} c_{4}\right)(2 k)^{-1}, \\
& t_{32}=\left(\frac{1+2 g c_{1} c_{2}}{G} \exp ^{-i k L}-\frac{2 g}{G^{2}}-\frac{\exp ^{i k L}}{G}-c_{1} c_{2} c_{4}\right)(2 k)^{-1} \text {, } \\
& t_{33}=1-\left(\frac{2 g c_{1} \exp ^{-i k L}+\exp ^{i k L}}{G}-c_{1} c_{4}\right)(2 k)^{-1}, \\
& t_{34}=\left(\frac{2 g c_{1} \exp ^{-i k L}+\exp ^{i k L}}{G}-c_{1} c_{4}\right)(2 k)^{-1}, \\
& t_{41}=-\left(\frac{2 g}{G}+c_{1} c_{2} c_{4}\right)(2 k)^{-1}, \\
& t_{42}=-\left(\frac{\exp ^{i k L}}{G}-\frac{2 g}{G^{2}}-c_{1} c_{2} c_{4},\right)(2 k)^{-1}, \\
& t_{43}=-\left(-\frac{\exp ^{i k L}}{G}+c_{1} c_{4},\right)(2 k)^{-1}, t_{44}=1+c_{1} c_{4}(2 k)^{-1} \text {. }
\end{aligned}
$$


Здесь $L-$ расстояние между $z_{12}$ и $z_{23}$ в продольном направлении, т. е. $x_{23}-x_{12}$,

$$
\begin{gathered}
c_{1}=\frac{G^{2}}{4 g\left(G^{2}-2 g^{2}\right)}, \quad c_{2}=\frac{4 g^{2}-G}{G}, \\
c_{3}=\frac{G^{2}-2 g^{2}}{G^{2}}, \quad c_{4}=\frac{4 g^{2}-G^{2}}{G^{2}} .
\end{gathered}
$$

Матрица $T$ есть матрица преобразования (матрица квантового вентиля) в нашем подходе. Нормированное расстояние $d$ от подпространства тензорных произведений находим численно. В рассматриваемой системе ситуация с реализуемой операцией зависит от волнового числа $k$. Эта зависимость показана на рис. 2. На графике выбраны безразмерные единицы. Все волноводы имеют одинаковую ширину $L=1$.

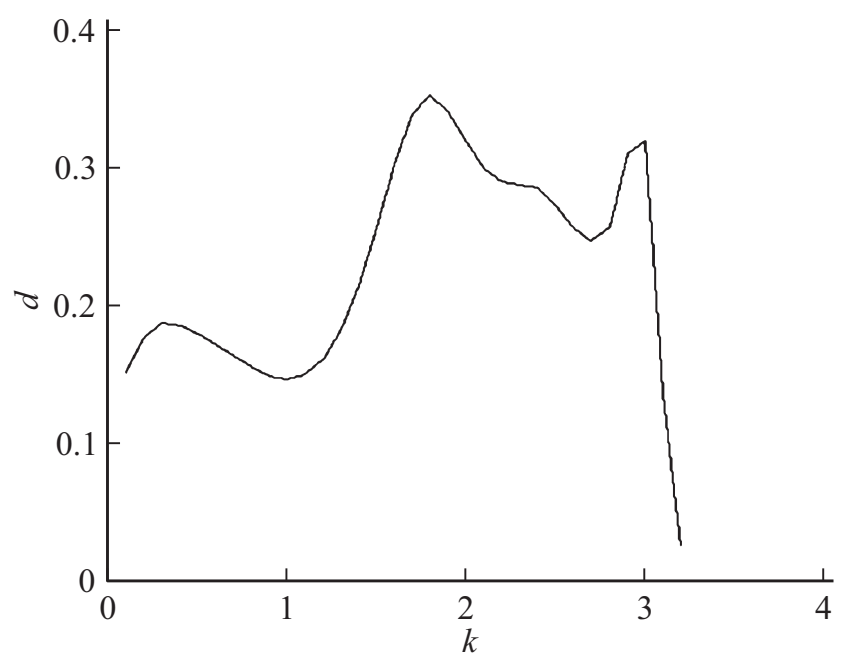

Рис. 2. Расстояние $d$ матрицы квантового вентиля до подпространства тензорных произведений как функция от волнового числа $k$ (единицы безразмерные): $k_{0}=i, L=1, x_{12}=x_{34}=0$, $x_{23}=2$.

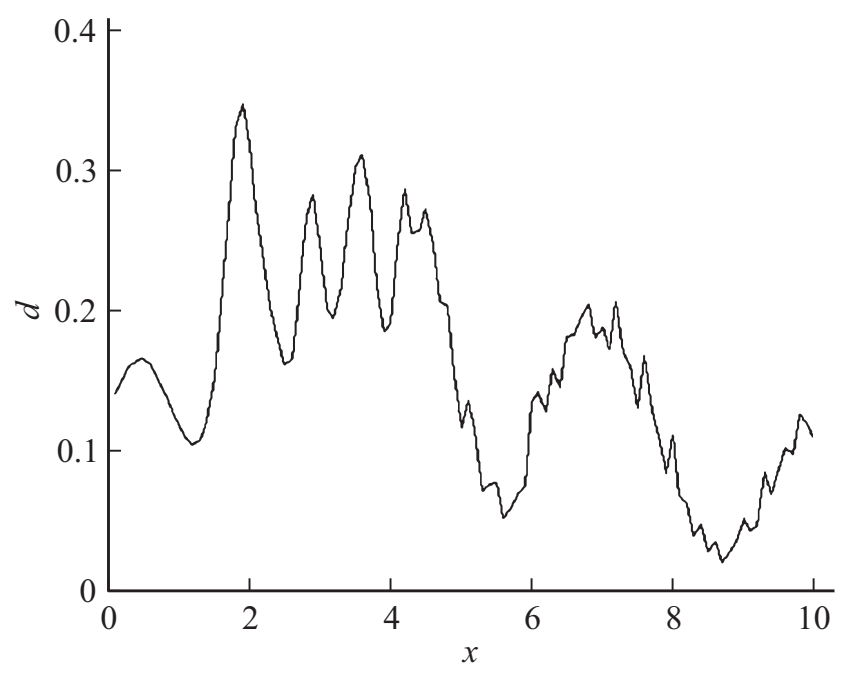

Рис. 3. Расстояние $d$ матрицы квантового вентиля до подпространства тензорных произведений как функция от расстояния $\mathrm{x}$ между отверстиями связи (единицы безразмерные): $k_{0}=i$, $L=1, x_{12}=x_{34}=0, x_{23}=x, k=2$.
Зависимость расстояния матрицы квантового вентиля до подпространства тензорных произведений от положения отверстия связи $x_{23}=x$ показана на рис. 3 . Ярко выраженные осцилляции показывают возможность управления степенью независимости преобразования кубитов за счет изменения параметров системы.

\section{Доказательство теоремы}

Построим матрицы $R, R, A$ в соответствии с описанной выше процедурой. Пусть vecB $(\text { vec } C)^{\prime}=k b c^{\prime}$, где $\left\|b_{m^{2} \times 1}\right\|=\left\|c_{n^{2} \times 1}\right\|=1, k-$ нормирующий множитель. Рассмотрим разложение по сингулярным числам для матрицы $A$ :

$$
A=\sum_{i=1}^{s} \sigma_{i} u_{i} v_{i}
$$

где $s=\operatorname{rank} A, \sigma_{1} \geq \sigma_{2} \geq \ldots \geq \sigma_{s}>0-$ сингулярные числа матрицы, расположенные в убывающем порядке, $u_{i}$ - ортонормированные векторы размера $m^{2},\left\|u_{i}\right\|=1$, $v_{i}$ - ортонормированные векторы размера $n^{2},\left\|v_{i}\right\|=1$. Тогда

$$
\rho=\left\|A-\operatorname{vec} B(\operatorname{vec} C)^{\prime}\right\|=\left\|\sum_{i=1}^{s} \sigma_{i} u_{i} v_{i}-k b c^{\prime}\right\| .
$$

Мы рассматриваем норму Фробениуса для матрицы $M$ :

$$
\|M\|_{F}=\sqrt{\sum_{i=1}^{m} \sum_{j=1}^{n} a_{i j}^{2}}
$$

которая имеет следующее известное свойство:

$$
\|M\|_{F}^{2}=\operatorname{tr}\left(M M^{\prime}\right)=\operatorname{tr}\left(M^{\prime} M\right),
$$

где $\operatorname{tr}(M)$ - след матрицы $M, M^{\prime}$ - транспонированная матрица $M$. Рассмотрим

$$
\begin{aligned}
& \rho^{2}=\left\|\sum_{i=1}^{s} \sigma_{i} u_{i} v_{i}^{\prime}-k b c^{\prime}\right\|^{2}=\operatorname{tr}\left(\left(\sum_{i=1}^{s} \sigma_{i} u_{i} v_{i}^{\prime}-k b c^{\prime}\right)\right. \\
& \left.\times\left(\sum_{i=1}^{s} \sigma_{i} u_{i} v_{i}^{\prime}-k b c^{\prime}\right)\right)=\operatorname{tr}\left(\sum_{i=1}^{s} \sum_{j=1}^{s} \sigma_{i} \sigma_{j} v_{i} u_{i}^{\prime} u_{j} v_{j^{\prime}}\right. \\
& \left.-k c b^{\prime} \sum_{i=1}^{s} \sigma_{i} u_{i} v_{i}^{\prime}-\left(\sum_{i=1}^{s} \sigma_{i} v_{i} u_{i}^{\prime}\right) k b c^{\prime}+k^{2} c b^{\prime} b c^{\prime}\right) \\
& =\operatorname{tr}\left(\sum_{i=1}^{s} \sigma_{i}^{2}-k c b^{\prime} \sum_{i=1}^{s} \sigma_{i} u_{i} v_{i}^{\prime}-\left(\sum_{i=1}^{s} \sigma_{i} v_{i} u_{i}^{\prime}\right) k b c^{\prime}+k^{2}\right) .
\end{aligned}
$$

Учитывая свойства следа матрицы, получаем

$$
\begin{aligned}
& \rho^{2}=\sum_{i=1}^{s} \operatorname{tr}\left(\sigma_{i}^{2}\right)-k \sum_{i=1}^{s} \sigma_{i} \operatorname{tr}\left(c b^{\prime} v_{i} u_{i}^{\prime}\right) \\
& -k \sum_{i=1}^{s} \sigma_{i} \operatorname{tr}\left(v_{i} u_{i}^{\prime} b c^{\prime}\right)+\operatorname{tr}\left(k^{2}\right)=\sum_{i=1}^{s} \sigma_{i}^{2} \\
& -k \sum_{i=1}^{s} \sigma_{i} \operatorname{tr}\left(c b^{\prime} v_{i} u_{i}^{\prime}\right)-k \sum_{i=1}^{s} \sigma_{i} \operatorname{tr}\left(v_{i} u_{i}^{\prime} b c^{\prime}\right)+k^{2} .
\end{aligned}
$$


Заметим, что $\operatorname{tr}\left(v_{i} u_{i}^{\prime} b c^{\prime}\right)=\operatorname{tr}\left(v_{i} u_{i}^{\prime} b c^{\prime}\right)=\operatorname{tr}\left(\left(b c^{\prime}\right)\left(v_{i} u_{i}^{\prime}\right)\right)=$ $=\operatorname{tr}\left(c b^{\prime} v_{i} u_{i}^{\prime}\right)$. Имеем

$$
\rho^{2}=\sum_{i=1}^{s} \sigma_{i}^{2}-2 k \sum_{i=1}^{s} \sigma_{i} \mathrm{t} r\left(c b^{\prime} v_{i} u_{i}^{\prime}\right)+k^{2} .
$$

Пусть $c=\left[c_{l}\right]_{l=1}^{n^{2}}, \quad b=\left[b_{j}\right]_{j=1}^{m^{2}}, \quad u_{i}=\left[u_{j}^{i}\right]_{j=1}^{m^{2}}, \quad v_{i}=\left[v_{l}^{i}\right]_{l=1}^{n^{2}}$. Тогда

$$
c b^{\prime} u_{i} v_{i}^{\prime}=\left[c_{l} v_{t}^{i} \sum_{j=1}^{m^{2}} b_{j} u_{j}^{i}\right]_{l, t=1}^{n^{2}} .
$$

Приходим к выражению

$$
\begin{aligned}
\rho^{2} & =\sum_{i=1}^{s} \sigma_{i}^{2}-2 k \sum_{i=1}^{s}\left(\sigma_{i} \sum_{l=1}^{n^{2}}\left(v_{l}^{i} c_{l}\right) \sum_{j=1}^{m^{2}}\left(b_{j} u_{j}^{i}\right)\right)+k^{2} \\
& =\sum_{i=1}^{s} \sigma_{i}^{2}-2 k \sum_{i=1}^{s} \sigma_{i}\left(\sigma_{i} v_{i}^{\prime} c b^{\prime} u_{i}\right)+k^{2} .
\end{aligned}
$$

Рассмотрим это выражение как функцию от $k$. Она имеет минимум при

$$
k=\sum_{i=1}^{s}\left(\sigma_{i} v_{i}^{\prime} c b^{\prime} u_{i}\right)
$$

Подставляя это значение в (3), получаем

$$
\sum_{i=1}^{s} \sigma_{i}^{2}-\left(\sum_{i=1}^{s}\left(\sigma_{i} v_{i}^{\prime} c b^{\prime} u_{i}\right)\right)^{2} .
$$

Это выражение минимально, если $\sum_{i=1}^{s} \sigma_{i}\left|v_{i}^{\prime} c b^{\prime} u_{i}\right|$ максимально. Из-за упорядоченности сингулярных чисел имеем

$$
\sum_{i=1}^{s} \sigma_{i}\left|v_{i}^{\prime} c b^{\prime} u_{i}\right| \leq \sum_{i=1}^{s} \sigma_{1}\left|v_{i}^{\prime} c b^{\prime} u_{i}\right| .
$$

Благодаря неравенству Гельдера получаем

$$
\left|v_{i}^{\prime} c\right|^{2} \leq\left\|v_{i}^{\prime}\right\|^{2}\|c\|^{2}=1 .\left|b^{\prime} u_{i}\right|^{2} \leq\left\|b^{\prime}\right\|^{2}\left\|u_{i}\right\|^{2}=1 .
$$

Значит, $\left|v_{i}^{\prime} c b^{\prime} u_{i}\right|=1$ для $c=v_{1}, b=u_{1} . \sigma_{1}-$ максимальное сингулярное число, поэтому $\sum_{i=1}^{s} \sigma_{1}\left|v_{i}^{\prime} c b^{\prime} u_{i}\right|$ максимально при $k=\sigma_{1}, c=v_{1}, b=\sigma_{1}$.

Итак, для заданной матрицы $A_{m n \times m n}$ норма $\|A-B \otimes C\|$ минимальна, если матрицы $B_{m \otimes m}$ и $C_{n \otimes n}$ выбраны так, что $\operatorname{vec} B(\operatorname{vec} C)^{\prime}=\sigma_{1} u_{1} v_{1}^{\prime}$.

Эта норма такова

$$
\|A-B \otimes C\|=\left\|A-\operatorname{vec} B(\operatorname{vec} C)^{\prime}\right\|=\left\|A-\sigma_{1} u_{1} v_{1}^{\prime}\right\| .
$$

Эта норма и дает расстояние от матрицы $A_{m n \times m n}$ до подпространства матриц, являющихся тензорными произведениями матриц размеров $m \times m$ и $n \times n$.

Работа частично поддержана Правительством Российской Федерации (грант 074-U01), грантом 16-1110330 РНФ

\section{Список литературы}

[1] Nielsen M.A., Chuang I.L. Quantum Computation and Quantum Information. Cambridge University Press, Cambridge, 2001.

[2] Herbst T., Scheidl T., Fink M., Handsteiner J., Wittmann B., Ursin R., Zeilinger A. // PNAS. 2015. V. 112. P. 14202.

[3] Gubaidullina K.V., Chivilikhin S.A. // Nanosystems: Phys. Chem. Math. 2017. V. 8. P. 243.

[4] Blinova I.V., Popov I.Y. // Phys. Elem. Particles Atomic Nucl. Lett. 2011. V. 8. P. 767.

[5] Miroshnichenko G.P., Trifanov A.I. // Quantum Inf. Process. 2013. V. 12. P. 1417.

[6] Holevo A.S. Quantum Systems, Channels, Information: a Mathematical Introduction. Walter de Gruyter $\mathrm{GmbH} \& \mathrm{Co}$. KG, Berlin, 2012.

[7] Liu C.L., Zhang D.-J., Yu X.-D., Ding Q.-M., Liu L. // Quantum Inf. Process. 2017. V. 16. P. 198.

[8] Vedral V., Plenio M.B., Rippin M.A., Knight P.L. // Phys. Rev. Lett. 1997. V. 78. P. 2275.

[9] Vedral V., Plenio M.B. // Phys. Rev. A. 1998. V. 57. P. 1619.

[10] Ozawa M. // Phys. Lett. A. 2000. V. 268. P. 15.

[11] Gilchrist A., Langford N.K., Nielsen M.A. // Phys. Rev. A. 2005. V. 71. P. 062310.

[12] Gisin N., Ribordy G., Tittel W., Zbinden H. // Rev. Mod. Phys. 2002. V. 74. P. 145.

[13] Gu S.-J. // Int. J. Mod. Phys. B. 2010. V. 24. P. 4371.

[14] Taddei M.M., Escher B.M., Davidovich L., de Matos Filho R.L. // Phys. Rev. Lett.2013. V. 110. P. 050402.

[15] Caruso F., Giovannetti V., Lupo C., Mancini S. // Rev. Mod. Phys. 2014. V. 86. P. 1203.

[16] Aolita L., de Melo F., Davidovich L. // Rep. Prog. Phys. 2015. V. 78. P. 042001.

[17] Streltsov A. Quantum Correlations Beyond Entanglement and Their Role in Quantum Information Theory. Springer, Berlin, 2015.

[18] Yu C.-S., Zhang Y., Zhao H. // Quantum Inf. Process. 2014. V. 13. P. 1437.

[19] Цуканов А.В. // Опт. и спектр. 2017. Т. 123. С. 591; Tsukanov A.V. // Opt. Spectrosc. 2017. V. 123. P. 602.

[20] Pittenger A.O., Rubin M.H. // Linear Algebra Appl. 2002. V. 346. P. 47.

[21] Dahl G., Leinaas J.M., Myrheim J., Ovrum E. // Linear Algebra Appl. 2007. V. 420. P. 711.

[22] Markovsky I. Low-Rank Approximation: Algorithms, Implementation, Applications. Springer, Berlin, 2012.

[23] Gohberg I.C., Krein M.G. Introduction to the Theory of Linear Nonselfadjoint Operators in Hilbert Space. Translations of Mathematical Monographs. AMS, Providence, RI, 1969.

[24] Peller V.V. Hankel Operators and Their Applications. Springer Monographs in Mathematics. Springer-Verlag, N.Y., 2003.

[25] Dinur I., Sudan M., Wigderson A. // Lect. Notes Comp. Science. 2006. V. 4110. P. 304.

[26] Gavrilov M., Gortinskaya L., Pestov A., Popov I., Tesovskaya E. // Phys. Elem. Particles Atomic Nucl. Lett. 2007. V. 4. P. 237.

[27] Popov I.Yu., Popova S.L. // Phys. Lett. A. 1993. V. 173. P. 484

[28] Popov I.Yu., Popova S.L. // Europhys. Lett. 1993. V. 24. P. 373.

[29] Popov I.Yu. // Z. Anal. Anwend. 2013. V. 32. P. 155.

[30] Albeverio S., Gesztesy F., Hoegh-Krohn R., Holden H. with an appendix by Exner $P$. Solvable Models in Quantum Mechanics. AMS Chelsea Publishing, Providence, RI, 2005. 